\title{
Statistical Analysis of Adult HIV/AIDS Patients and Modelling of AIDS Disease Progression
}

\author{
Desalegn Petros Kelkile \\ Department of Statistics, Dilla University, Dilla Town, Ethiopia
}

\section{Email address:}

desye.peter@gmail.com

\section{To cite this article:}

Desalegn Petros Kelkile. Statistical Analysis of Adult HIV/AIDS Patients and Modelling of AIDS Disease Progression. Science Journal of Applied Mathematics and Statistics. Vol. 4, No. 5, 2016, pp. 189-201. doi: 10.11648/j.sjams.20160405.12

Received: August 4, 2016; Accepted: August 13, 2016; Published: September 13, 2016

\begin{abstract}
This study has been intended to apply the cox proportional hazard model to review the determinant factors of survival time and discrete time homogeneous semi markov model to predict the clinical progression of AIDS disease by secondary data obtained from the antiretroviral therapy unit of Dilla and Hawassa University Referral Hospitals. Patients were followed for a median of 34 months. Of total sample, 517 (68.4\%) were female and $239(31.6 \%)$ were male. In the followed up period, $110(14.5 \%)$ patients died and $646(85.5 \%)$ patients were censored. The cox regression result indicated that the survival time of the HIV patient was significantly connected with adherence level, age, alcohol use, CD4, condom use, functional status, marital status and WHO stage. The outcome of homogenous semi-markov model showed that the survival probability of a patient increased when CD4 count increased. The study is suggested that the above significant variables should be viewed as significant component of the routine clinical care for patients on ART and patients require checking CD4 count in the suitable day as physician arrange to know their disease stage.
\end{abstract}

Keywords: Antiretroviral Therapy, Cox Proportional Hazard Model, Discrete Time Homogeneous Semi-Markov Model, AIDS Progression

\section{Introduction}

\subsection{Background}

According to the 2007 account of the FMOH [7], AIDS epidemic is one of the most unhelpful epidemics in the history of human kind, claiming the lives of people. Since the recognition of the disease, HIV has infected close to 71 million people, and more than 30 million have died due to AIDS till 2007. More than $66 \%$ of the people living with HIV/AIDS (PLWHA) are in Sub-Saharan Africa, where AIDS is the leading cause of death. However, newly new HIV infection is declining. In 33 countries, HIV incidence has fallen by more than $25 \%$ between 2001 and 2009. Of these countries, 22 are in sub-Saharan Africa. The biggest epidemics in Sub-Saharan Africa Ethiopia, Nigeria South Africa, Zambia and Zimbabwe have either stabilized or are presentation signs of declines [17].

\section{HIV/AIDS in Ethiopia}

Ethiopia is a large, predominantly rural, country covering an area of 1.13 million sq $\mathrm{km}$. The country is a Federal
Democratic Republic, with a decentralized administrative structure, composed of nine regional states and two city administrations. Ethiopia is currently the second most populous country in Africa (next to Nigeria) with a total population of about 79 million, increasing at an annual rate of $2.6 \%$, according to the 2007 national census [8].

The first time that HIV observed in Ethiopia was in 1984 and the first two AIDS cases were reported in 1986. Then, National Aids Control Program (NACP) was established at the department level at the $\mathrm{MOH}$ in 1987 [7].

In 2005 , the estimated national HIV prevalence was 3.5\% (10.5\% for urban and $1.9 \%$ for rural areas) according to Federal Ministry of Health report [7]. In 2010, in Ethiopia, there are 1.2 million people living with HIV (PLHIV), with an adult HIV prevalence of $2.4 \%$ (7.7\% urban and $0.9 \%$ rural) and male-female ratio of $1.9 \%$. A total of 397,818 people living with HIV are estimated to be in need of antiretroviral treatment (ART), and an estimated 137,494 new HIV infections are expected to join in the given year. The HIV/AIDS epidemic in Ethiopia is generalized with significant heterogeneity between regional states and 
population groups [7].

According to Ministry of Health [8], the three highest prevalence regions in the country in 2010 are Gambela $(8.3 \%$ urban), Addis Ababa (7.5\%) and Dire Dawa (4.2\%). Other regions with HIV prevalence rates greater than the national estimate $(2.1 \%)$ are Harari $(3.2 \%)$, Amhara $(2.7 \%)$ and Tigray (2.7\%). Somali is the region with the lowest HIV prevalence estimate in the country $(0.8 \%)$. By the year 2007, in UNAIDS report [17], the number of PLWHIV requiring ART was 258,264 . Of these, 242,548 were adults $(24.8 \%$ of those 15 years and older living with HIV) and 15,716 were children ( $24.3 \%$ of HIV positive children in the age of 0 to 14 years).

Overview of ART

Anti-Retroviral Therapy (ART) is the administration of at least three different medications known as Anti-Retroviral drugs (ARV) in order to suppress the replication of the human immunodeficiency virus (HIV) [14]. Antiretroviral (ARV) drugs can reduce the ability of the virus to replicate, and they can thus reduce the damage that the virus does over time on the patient's body. ART is delivered as part of a comprehensive care, which includes Voluntary Counseling and Testing (VCT), the diagnosis and treatment of Sexually Transmitted Diseases (STDs), Tuberculosis (TB), Opportunistic Infections (OI), and the prevention of mother to child transmission (PMTCT) as well as the treatment of pregnant women.

The effect of antiretroviral therapy are especially evident in sub saharan Africa, where an estimated 320000 (or 20\%) fewer people died of AIDS related causes in 2009 than in 2004, when antiretroviral therapy began to be dramatically expanded [17].

Antiretroviral therapy began in 2003 and free ART was launched in Ethiopia in January of 2005, and the Accelerating Access to HIV/AIDS Treatment in Ethiopia, Road map 20042006 in June of 2005. The Government of Ethiopia issued the first antiretroviral (ARV) guidelines in 2003, which were revised in 2005 to facilitate a rapid scale up of ART. The Road map targets to put 100,000 persons on ART by the end of 2006. By the end of July 2006, 45,595 patients had ever started on ART at 132 facilities across the country. Of these, 35,460 were on treatment currently and 18,384 were enrolled in the first six months of 2006 [5].

In Oromiya region, 63,931 patients were requiring ART in 2005. This study started when Federal $\mathrm{MOH}$ was planning to realize universal access to free ART [7]. Successful implementation of such initiative needs also quantitative statistical analysis in appropriate setting. Currently ART is given at national level in public hospitals, health centers and private hospitals under the support of federal ministry of health. This study will answer what are the determinants of HIV/AIDS patients' survival time and what looks like the clinical progression of AIDS disease.

It is hoped that this research serve will as an input for policy makers, guide line for implementation of ART at national level and concerned health specialists who work on providing care, support and treatment aspect of the HIV/AIDS programs in the country.

\subsection{Statement of the Problem}

Although ART is delivered as part of a comprehensive care with emphasis on the reduction in HIV related morbidity and mortality, the observed changes are not sufficient enough compared to the desired goals of the ART, especially, in developing countries like Ethiopia where survival of HIV/AIDS patients in ART depends on a variety of factors, which may also vary greatly with economic, demographic, behavioral risk and health factors.

HIV/AIDS is affecting every sector of the Ethiopian society that found at macro level, agriculture, education, health, business and industry sectors. All are adversely impacted by the disease. To reduce HIV/AIDS related mortality, Ethiopian free ART service has been expanding in the past five years but there is limited information regarding treatment outcome and determinant factors in patient survival time. Most study at national level focuses on the prevention and on factors that increase the chance of contracting the disease. However, the determinant factor of survival for People living with Hiv/Aids and the clinical progression of AIDS disease are not well studied yet. Studies addressing those issues will contribute in strengthening the implementation of ART.

In this research, efforts have been made to answer some of the questions that are remained unanswered regarding the survival analysis of HIV/AIDS patients:

1. What are the determinant factors of survival time for HIV/AIDS adult patients?

2. What is the probability of a patient going a worse stage and to death form any AIDS disease stage?

3. What is the probability of remaining or leaving the baseline clinical AIDS disease stage of an individual in the given time?

4. What is the probability of a patient to be alive in the given interval of time from any clinical AIDS disease stage?

\subsection{Objectives of the Study}

General Objective:

The main objective of this study is to investigate the determinants of survival time among adult HIV/AIDS patients and stochastic modelling of AIDS disease progression, using the data obtained from Hawassa and Dilla University Referral Hospital ART centers.

Specific Objectives

1. To assess the five year survival probability pattern of HIV/AIDS patients on ART.

2. To estimate and compare survivorship function in subgroups within variable category.

3. To fit cox proportional hazard regression model and determine the significant predicators of survival time of HIV/AIDS patients.

4. To fit the discrete time homogeneous semi-markov model including its reliability and failure rate model, to predict individual clinical progression of AIDS disease.

5. To provide quantitative information from this study to policy makers and researchers. 


\section{Methodology}

Data: The data for this study were collected from Hawassa and Dilla University Referral Hospital ART center, Ethiopia. The study is retrospective study and has considered the HIV/AIDS patients in antiretroviral therapy (ART) unit of Hawassa and Dilla University Referral Hospital from September 2007 up to August 2012.

Samling Technique: In this study Simple random sampling method was adopted as an appropriate sampling design for selecting a representative sample of the adult HIV/AIDS patients based on their Referral Hospital ART center (Hawassa and Dilla University).

Sample Size: the required sample size for this study was 756 patients from a total of 5500 adult HIV/AIDS patients in Hawassa and Dilla University Referral Hospital ART center between 2007 and 2012 G.C.

About Methodology: Survival Analysis and Stochastic were applied to investigate the determinants of survival time among adult HIV/AIDS patients and stochastic modelling of AIDS disease progression, using the data obtained from Hawassa and Dilla University Referral Hospital ART centers.

\section{Survival Analysis}

Survival analysis involves the modeling and analysis of data having a principal end point, the time until an event occurs (time-to-event data). Such types of data frequently arise from medicine, public health and demography where the analysis is usually referred to as Survival data analysis and industrial studies in engineering fields as reliability analysis.

Survival time is the duration of time till an event occurs. The inherent aging process that is present when subjects are followed over time is what distinguishes survival time from other dependent variables. Start and end of survival time depends on the context of the problem under investigation. For example, the lifetimes of machine components in industrial reliability, if the end point of a patient is death in medicine, the durations of strikes or periods of unemployment in economics, the time taken by subjects to complete specified tasks in psychological experimentation, the lengths of tracks on a photographic plate in particle physics, the performance of a certain task in a learning experiment in psychology or a change of residence in a demographic study and the survival times of patients in a clinical trial.

Survival data could be incomplete due to censoring and truncation. Censoring is a form of missing data, problem which is common in survival analysis or when we don't know the survival time exactly. A censored observation is one whose value is incomplete due to random factors for each subject.

Right censoring:

It happens when a subject's follow-up time terminates before the outcome of interest is observed. This is the most common form of censoring for incomplete data. The presence of censoring in the data makes the study of survival time more interesting from a statistical research perspective. The sample data intended for this research will encounter one of the most common types of censoring called right censoring for the reason that individuals might survive beyond the study period, lost to follow-up, dropped out from the study and also might die due to the disease other than HIV. It particularly follows a right random censoring mechanism since the patient's entry into the study period is random. The failure time is the same as the exact failure time when an event occurs, and censored time is the difference between the time when an event occurs and patients' enrollment in a study.

\subsection{Survival Function and Hazard Function}

In summarizing survival data there are two functions of central interest, namely, the survivor function and the hazard function.

$\mathrm{T}$ is a failure time (survival time, life time) non negativevalued random variable. The value of $\mathrm{T}$ for this study will be the time from start of treatment up to an event (i.e. death or censored) occurs.

The survivor function gives the probability that a person survives longer than some specified time $t$ and denoted by $\mathrm{S}$ $(t)$. survivor function $(S(t))$ gives the probability that $T$ exceeds the specified time $t$. now suppose that the random variable $\mathrm{T}$ has a cumulative probability distribution $F(t)$ with underlying probability density function $f(t)$. The survival function $S(t)$ is then given by:

$$
S(t)=\operatorname{Pr}(T \geq t)=1-\operatorname{Pr}(T<t)
$$

Cumulative distribution function $\mathrm{F}(\mathrm{t})$ is given as: $F(t)=$ $\operatorname{Pr}(T \leq t)$, then the survival function

$$
\begin{aligned}
S(t) & =P(T>t)=\int_{t}^{\infty} f(u) d u \\
& =1-\int_{0}^{t} f(u) d u \\
& =1-F(t)
\end{aligned}
$$

Characteristics of survival function $S(t)$ are:

- $\mathrm{S}(\mathrm{t})=1$ if $\mathrm{t} \leq 0, \mathrm{~S}(\infty)=\lim _{\mathrm{t} \rightarrow \infty} \mathrm{S}(\mathrm{t})=0$

- $\mathrm{S}(\mathrm{t})$ is non-increasing in $\mathrm{t}$

- $\mathrm{S}(\mathrm{t})$ is left continues

The hazard function that is denoted by $h(t)$ gives the instantaneous rate of failure, given that the individual has survived up to time $t$. The hazard function is also known as the conditional failure rate or simply hazard rate and is defined as the probability that an individual fails at time $t$, conditional on the fact that he or she has survived to that time. It therefore, represents the instantaneous failure rate for an individual surviving to time $t$. For $h(t) \geq 0$, the hazard function $h(t)$ is given by the following:

$$
\begin{gathered}
h(t)=P(T=t \mid T \geq t) \\
=\frac{P(T=t)}{P(T \geq t)}=\frac{f(t)}{s(t)}
\end{gathered}
$$

Let $\mathrm{H}(\cdot)$ be the cumulative hazard function.

$$
H(t)=\int_{0}^{t} h(u) d u
$$


The survival function in terms of the hazard function is given by

$$
S(t)=e^{-\int_{0}^{t} h(u) d u}=e^{-H(t)}
$$

The probability density function in terms of the hazard function can also be derived

$$
f(t)=h(t) \exp \left(\int_{0}^{t} h(u) d u\right)
$$

The mean survival time is given as:

$$
E(T)=\int_{0}^{\infty} t f(t) d t=\int_{0}^{\infty} S(t) d t
$$

The median survival time given as:

$$
S\left(t_{\text {med }}\right)=0.5, t_{\text {med }}=S^{-1}(0.5)
$$

\subsection{Non-parametric Estimator of Survival Function}

\section{Kaplan-Meier Estimator}

These methods are said to be non-parametric or distribution-free since they do not require specific assumption to be made about the underlying distribution of the survival times. For this study, random right censoring and independent censoring were applied.

The Kaplan-Meier (KM) estimator, also known as the product limit estimator, is the estimator used by most software packages. It incorporates information from all of the observations available, both censored and uncensored, by considering any point in time as a series of steps defined by the observed survival and censored times. When there is no censoring, the estimator is simply the sample proportion of observations with event times greater than $t$. The technique becomes a little more complicated but still manageable and interesting when censored times are included.

Assume we have a sample of $n$ independent observations, their survival times denoted by $t_{1}, t_{2}, \ldots, t_{n}$ and indicator of censoring by $\delta_{1}, \delta_{2}, \ldots, \delta_{\mathrm{n}}$ where $\delta_{\mathrm{i}}=1$, event/death and $\delta_{\mathrm{i}}=$ 0 for censored observations. The survival data are denoted by $\left(\mathrm{t}_{\mathrm{i}}, \delta_{\mathrm{i}}\right), \mathrm{i}=1,2, \ldots, \mathrm{n}$. The first step to obtain the KaplanMeier estimator of the survival function is to order the survival times as $\mathrm{t}_{(1)}<\mathrm{t}_{(2)} \ldots<\mathrm{t}_{(\mathrm{n})}$. Assume that among the $n$ observations there are $m \leq n$ failures occurred at distinct $m$ times. Then the rank- ordered failure times $\mathrm{t}_{(1)}<\mathrm{t}_{(2)} \ldots<\mathrm{t}_{(\mathrm{m})}$.

Define: $d_{(j)}=$ number of failure or death at time $t_{(j)}$

$\mathrm{N}_{(\mathrm{j})}=$ number of individual at risk at $\mathrm{t}_{(\mathrm{j})}$

The formula for Kaplan-Meier estimator of survival function at time $\mathrm{t}$ is given as:

$$
\begin{aligned}
& \hat{S}_{k m}(t)=\left(1-\frac{d_{(1)}}{N_{(1)}}\right)\left(1-\frac{d_{(2)}}{N_{(2)}}\right) \ldots\left(1-\frac{d_{(j-1)}}{N_{(j-1)}}\right) \\
= & \prod_{t(j)<t}^{k}\left(1-\frac{\mathrm{d}(\mathrm{j})}{\mathrm{N}(\mathrm{j})}\right) \text { for } t_{(k)} \leq t<t_{(k+1)}, k=1,2,2, \ldots, m
\end{aligned}
$$

When $\mathrm{t}=0, \mathrm{~S}(0)=1$, this means that all subjects alive at time 0 .

The variance of the Kaplan-Meier estimator of the survival function which is known as the greenwood formula is given as:

$$
\widehat{\operatorname{var}}(S(t))=[\hat{S}(t)]^{2} \sum_{t_{(i) \leq t}} \frac{d_{i}}{n_{i}\left(n_{i}-d_{i}\right)}
$$

The (1-a) X 100\% confidence interval for the survival function $\left(\mathrm{S}_{\mathrm{km}}(\mathrm{t})\right)$ at time $\mathrm{t}$ is:

$$
\hat{S}_{k m}(t) \pm Z_{\frac{\alpha}{2}} S . e\left(\hat{S}_{k m}(t)\right)
$$

The survival function changes only at failure times. That means decreasing step function takes a jump at failure times and also for censored times the conditional probability of survival is always 1.00. As a result of this, the survival probability is unchanged from the previous survival probability.

\subsection{Modelling of AIDS Disease Progression}

In this study, discrete time homogeneous semi-markov model was adopted for predicting the clinical progression of AIDS disease and the survival probability of an infected patient up to specified t month. This model was employed by Zelalem (2010) on data which was obtained from Feleg Hiwot hospital, Bahir-Dar. Patients were classified based on their immunological marker: state I ( CD4 $>$ 500 cells $\left./ \mathrm{mm}^{3}\right)$, state II $\left(350\right.$ cells $/ \mathrm{mm}^{3}<C D 4 \leq$ 500 cells $\left./ \mathrm{mm}^{3}\right)$, state III $\left(200\right.$ cells $/ \mathrm{mm}^{3}<$ CD4 $\leq 350$ cells $/ \mathrm{mm}^{3}$ ) and state IV (CD4 $\leq 200$ cells $\left./ \mathrm{mm}^{3}\right)$. Patient can go to any state at any time.

Immunological assessment using the CD4 count identifies the disease progression and determines initiation of treatment. CD4 testing can monitor the ART response as well (FMOH, 2007). In adult and adolescence, the WHO guideline recommend that ART be initiated in patients with history of an AIDS defining illness (CD4 cells $<200$ cells $/ \mathrm{mm}^{3}$ ) or CD4 cells count of less than 350 cells $/ \mathrm{mm}^{3}$. However, when CD4 is less than 200 cells $/ \mathrm{mm}^{3}$ delay in initiating ART may predispose the patient to other life threatening opportunistic infections. The optimum time to initiate ART is when a patient CD4 cell count is 200-350 (FMOH, 2007). The decision to start therapy or not in patients with CD4 count 350-500 depends on the potential benefits and risks of therapy, comorbidities, and patient readiness and willingness to adhere to long term treatment (Michael, 2010). In studies Europe and united states, patients who initiated ART at CD4 cell count greater than 350 cells/mm3 were more likely to have a normal CD4 cell count (Europe, $>800$ cells $/ \mathrm{mm}^{3}$; united states, $>500$ cells $/ \mathrm{mm}^{3}$ ) after several years of therapy than those who initiated ART at CD4 cell count less than 350 cells/mm3 (Michael, 2010).

Semi-Markov supposes that the probability of a patient going from one state to another does not depend on the past states where a patient has been, but depends on the current state. Moreover, probability that a patient jump from one state to another depends on the elapsed time in the current state. The model needs raw data obtained from observations, without strong assumptions about any standard probability functions regarding the random variables analyzed. Generally, the results are often very useful for physicians to make decisions. And, this study gives the probability of transition from one state to another by depending on the 
current state and the time elapsed in the current state. Finally, this study showed the clinical progression of AIDS disease.

\subsection{Semi-Markov}

A semi-Markov process is one that changes states in accordance with a Markov chain but takes a random amount of time between changes. More specifically, consider a stochastic process with state $1,2 \ldots \mathrm{N}$. The transition from state to state occurs at every time. The probability that a patient going from state $i$ to state $j$, given that the time until the transition from $i$ to $j$ occurs has distribution $F_{i j}$ is given by $P_{i j}, i, j>0$. Now, If we let $Z(t)$ denote the state at time $t$, then $\{\mathrm{Z}(\mathrm{t}), \mathrm{t}>0\}$ is called a semi-Markov process in (Bickel, et al, 2008). In predicting the future not only would we want to know the present state, but also the length of time that has been spent in that state.

\section{Results and Discussions}

\subsection{Descriptive Analysis}

This research has taken 756 HIV/AIDS patients who were followed up during the time from 2007 to 2012 at Hawassa and Dilla University Referral Hospital ART center. Of these $517(68.4 \%)$ were female and $239(31.6 \%)$ were male. The accounted death and censored of patients in the study period was $110(14.5 \%)$ and $646(85.5 \%)$, respectively. Since patients might survive beyond the study period, lost to follow up and might die due to other causes, the observations follow right censoring mechanism, random type in particular. The estimated mean survival time of the patient was 53.9 month with $95 \%$ confidence interval that as high as 55.7 month and as small as 52 month in Table 1. In Figure 1A, patients in ART were followed up for a median of 34 month (interquartile range 22-42 month). As we have seen in the Figure 1B, female patients in ART were followed up for a median survival time 33 month (interquartile range 22.2 - 43.7 month) while male patients followed up for a median survival time 34 month (interquartile range 23.0 - 41.7 month). Figure 2A represents the Kaplan-Meier estimator of the survivorship function for HIV/AIDS patients who followed up from 2007-2012 at Hawassa and Dilla University Referral Hospital ART center. The survival probability that a patient would live above 62 month, is 0.75 . The survivorship function estimate descends for the first 15 months quickly and then sharp tails off gradually, reaching its minimum value of 0.75 at 62 month. And, the initial steep descent shows that there were many patients who died shortly after initiation of ART. Figure 2 shows the hazard function goes up till to reach the minimum value 0.25 at 62 month. But, at the initial, the curve goes up sharply, but later the sharp tail is off gradually. The median age for patients, who had been in ART in 2007-2012 year, was 30 year (interquartile range 23-37 year). Of the 756 patients included, 609 (80.5\%) were from urban and 147 (19.5\%) were from rural in Table 1, This suggests that most of HIV/AIDS patients at Hawassa and Dilla University Referral Hospital ART center come from urban.

\section{Boxplot of survival time}

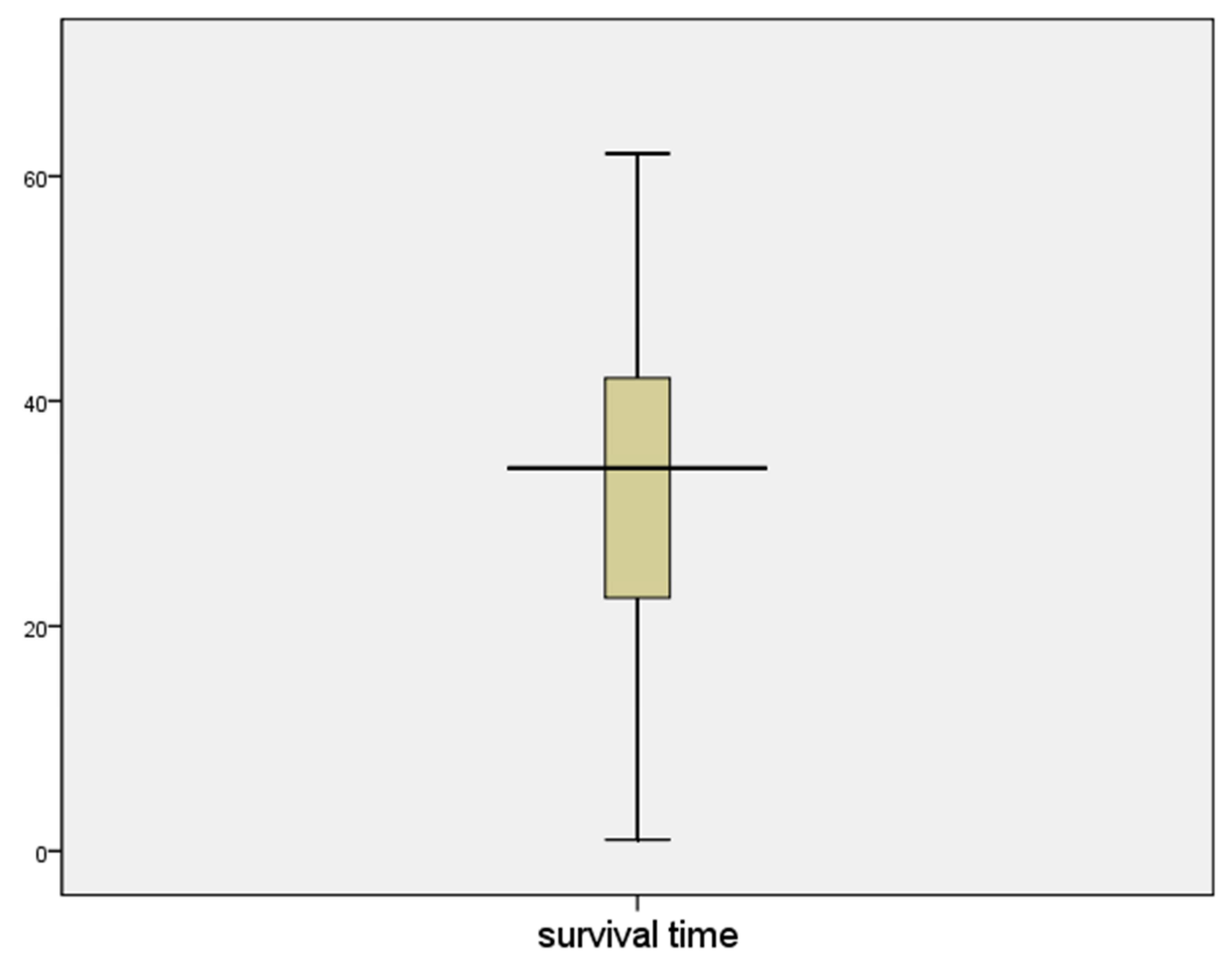




\section{Boxplots of time by Gender}

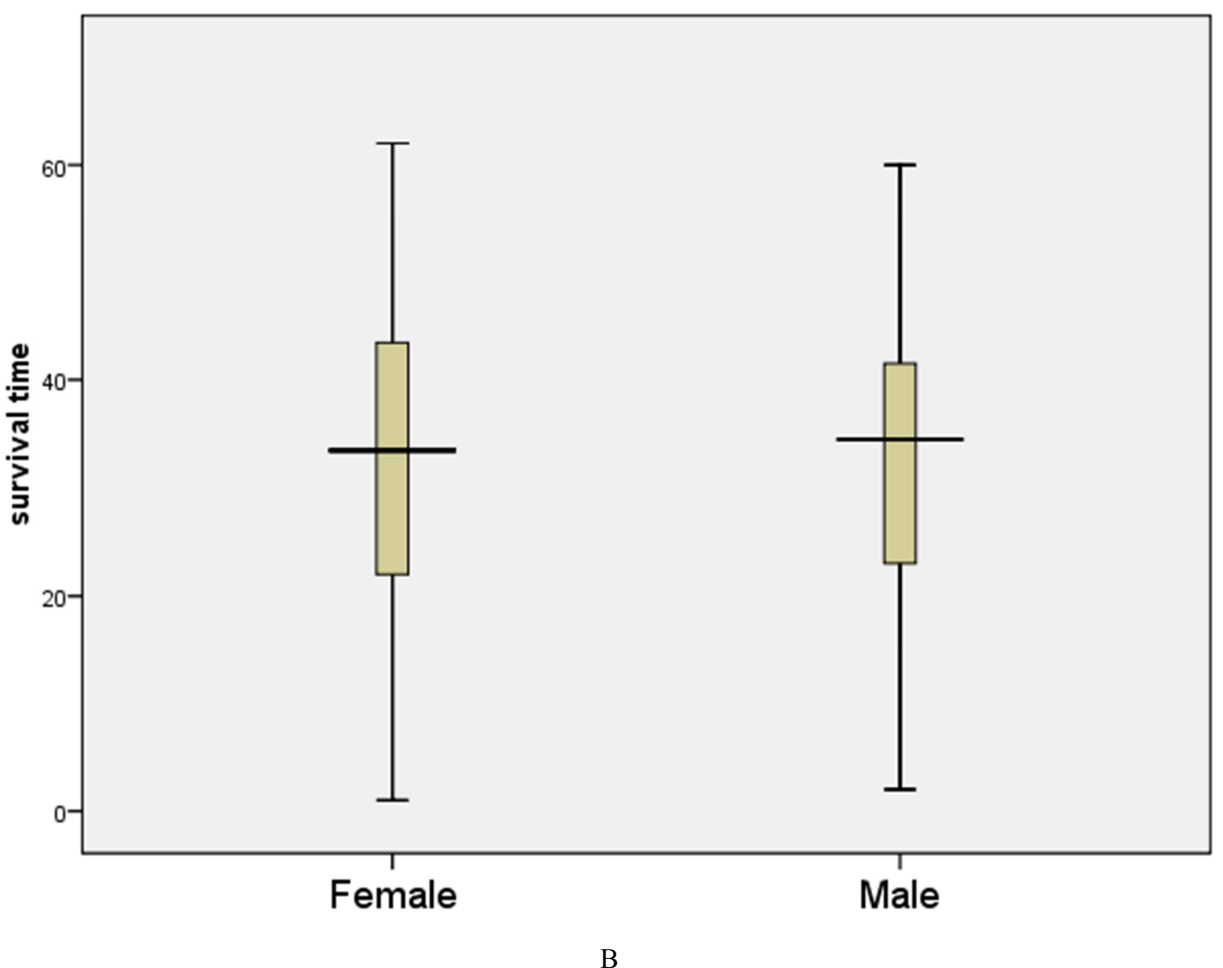

Figure 1. Boxplots A) Boxplot of Survival Time of HIV/AIDS Patients at Hawassa and Dilla University Referral Hospital ART Center B) Boxplots of Survival Time by Gender.

Table 1. Survival Status and Mean Survival Time for Adult HIV Positive Patients at Hawassa and Dilla University Referral Hospital.

\begin{tabular}{|c|c|c|c|c|c|c|}
\hline \multirow{2}{*}{ Death } & \multirow{2}{*}{ Censored } & \multirow{2}{*}{ Total } & \multirow{2}{*}{ Mean of survival time } & \multirow{2}{*}{ Std. Error } & \multicolumn{2}{|c|}{ 95\% C.I } \\
\hline & & & & & Lower & upper \\
\hline $110(14.5 \%)$ & $646(85.5 \%)$ & 456 & 53.93 & 0.917 & 52.132 & 55.724 \\
\hline
\end{tabular}

Survival Function

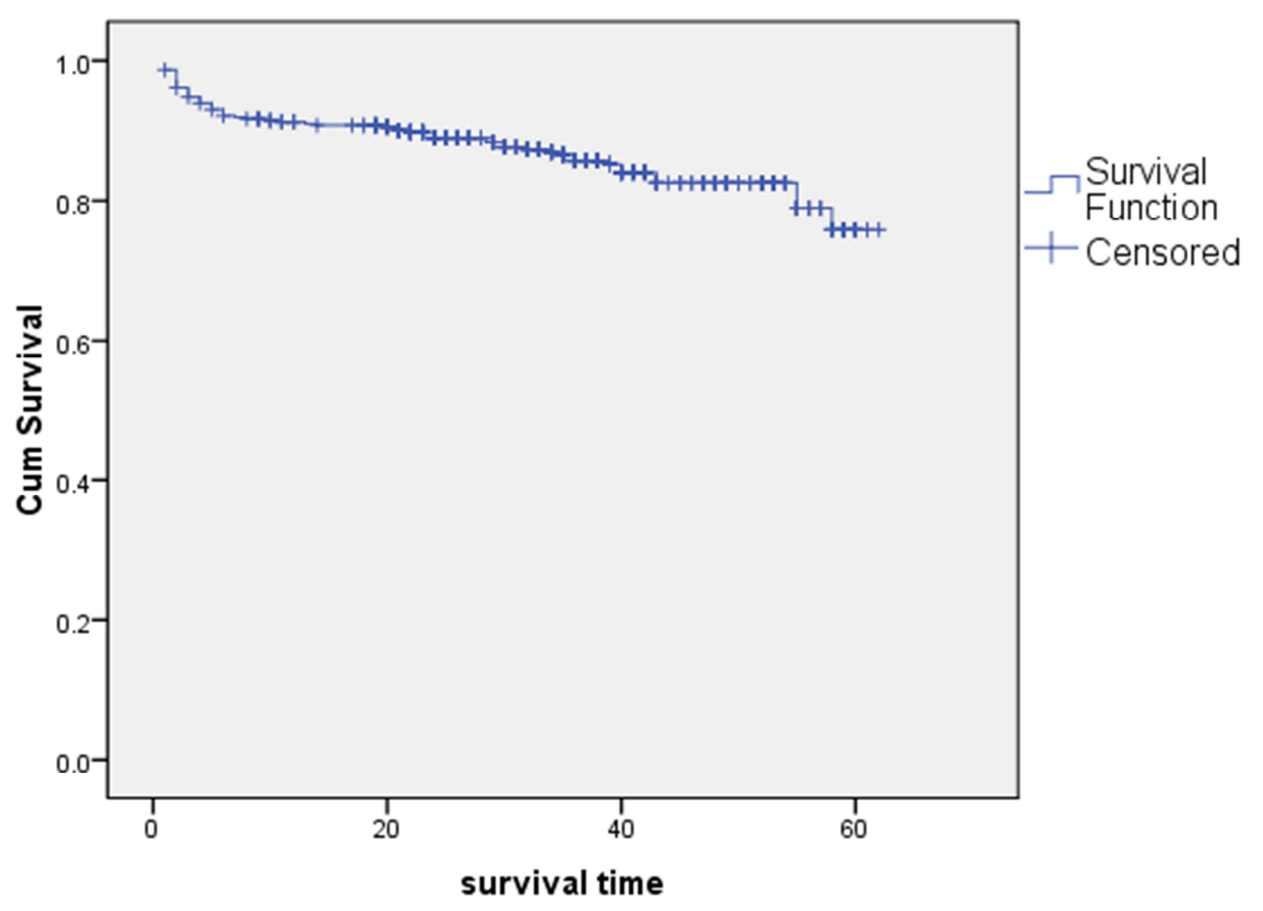




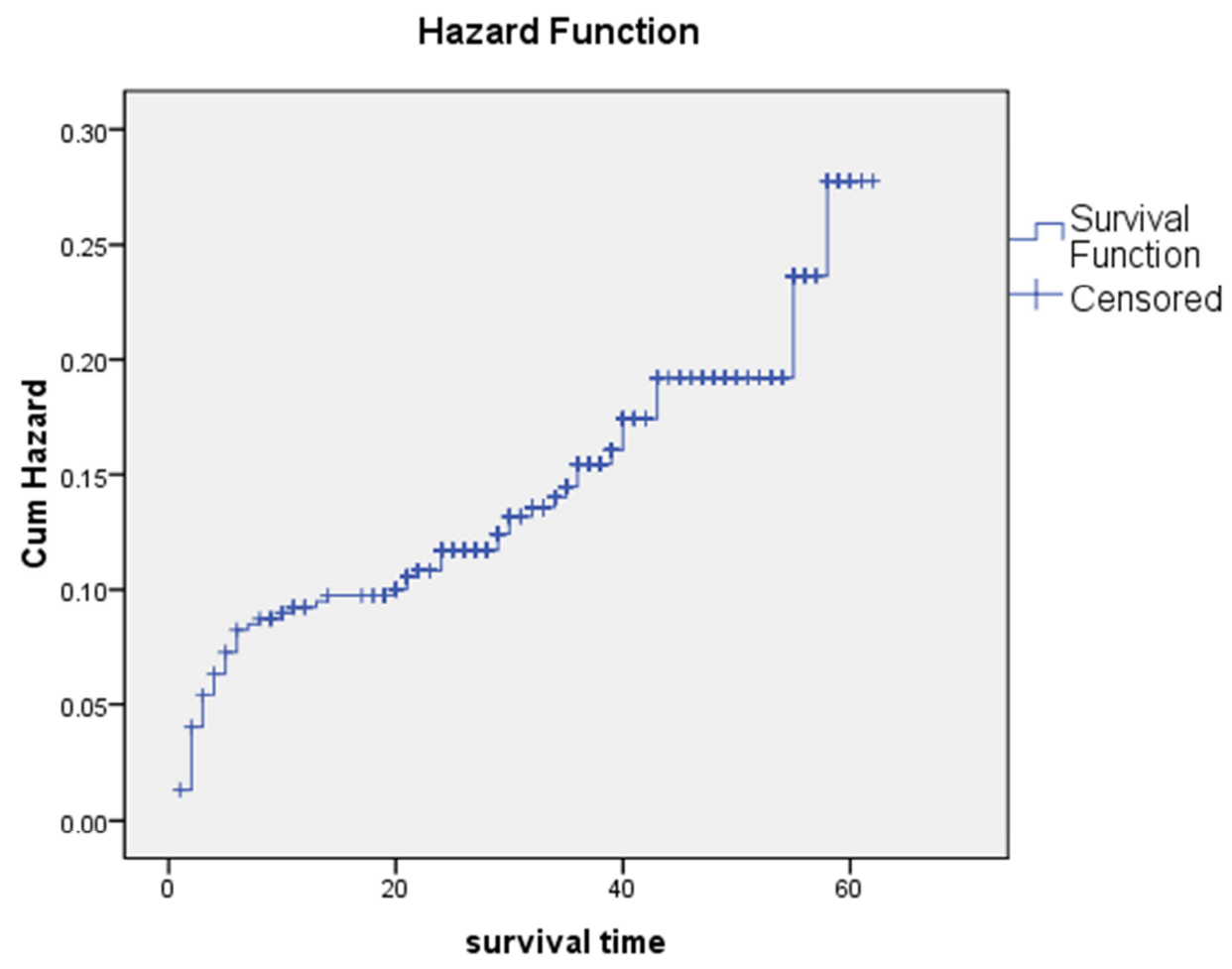

B

Figure 2. Estimated Survivorship Function and Hazard Function of HIV/AIDS Patients at Hawassa and Dilla University Referral Hospital HIV/AIDS ART Center for year 2007-2012.

\subsection{Inferential Analysis}

\subsubsection{Interpretation of Cox Regression Results}

The final model that is presented in Table 2 contains six categorical covariates (marital status, alcohol, condom use, functional status, WHO stage, and adherence level) and two continues covariates (CD4 cell count and age of the patient). Here, when we refer to "the model" we are referring to the one in Table 2. The estimated hazard ratio for a 1 year growth in the age of a patient is 1.050 . The interpretation is that the hazard of death increase by $5 \%$ for 1 year growth in age. The 95 percent confidence interval indicates that the rate could actually be as much as 8 percent or as little as 2 percent higher. Moreover, the interval estimate of hazard ratio for 5 year growth in the age of a patient is $\exp (5 * 0.049)=1.28$. This means that the hazard of death increase by $28 \%$ for 5 year growth in age. The estimated 95 percent confidence interval is $(1.11,1.46)$. This implies that for 5 year increase in the patient age, the hazard rate could be as much as 46 percent or as little as 11 percent higher.

For a 10 cells $/ \mathrm{mm}^{3}$ increase in CD4 count, the estimated hazard ratio is $\exp (10 *-0.004)=0.96$. This means that the patient whose increase the CD4 count by 10 cells $/ \mathrm{mm}^{3}$ may die at a rate which is less than 4 percent than the baseline CD4 count where a patient has been. The estimated confidence interval for hazard ratio is $(0.94,0.98)$, which implies that for every 10 cells $/ \mathrm{mm}^{3} \mathrm{CD} 4$ count increase in the HIV/AIDS patient the hazard rate is as much as 5 percent lower to 2 percent lower.

The reference category for covariate functional status was 'work'. The hazard ratio of bedridden HIV/AIDS patient was about 2.9, indicates that the hazard rate of bedridden patient is 2.9 times greater than patients who work. The estimated 95 percent confidence interval hazard ratio is $(1.06,8.17)$, this implies that the hazard ratio is as high as 8.17 and as low as 1.06. The hazard ratio of ambulatory HIV patient was 2.9, same as bedridden patients. The 95 percent hazard ratio confidence interval was $(1.6,5.3)$, this suggests that the hazard rate of ambulatory HIV positive patients is as much as 5.3 times higher or as little as 1.6 times higher than patients who could work. Moreover, we need to see the relationship between bedridden patient and ambulatory patient. To see their hazard ratio we need to conduct some hypothesis. Now, the null hypothesis says there is no significant difference between bedridden patients and ambulatory patients. Let $\beta_{1}$ and $\beta_{2}$ be the coefficient of bedridden patient and ambulatory patient, respectively. The hypothesis that going to be tested is the following:

$$
\text { Ho: } \beta_{1}=\beta_{2} \text { versus } H_{1}: \beta_{1} \neq \beta_{2}
$$

The test statistic is given as follows:

$$
\begin{gathered}
Z=\frac{\hat{\beta}_{1}-\hat{\beta}_{2}}{s \cdot e\left(\hat{\beta}_{1}-\hat{\beta}_{2}\right)} \\
Z=\frac{0.52-0.302}{0.516}=0.373
\end{gathered}
$$

The absolute value of $\mathrm{Z}$ is less than $Z_{0.025}=1.96$. This implies that the null hypothesis is not rejected. There is no 
statistical difference between bedridden patients and ambulatory patients.

The reference category for covariate adherence level is 'high adherence'. The estimated hazard ratio for patient with moderate adherence is 2.3 . Patients with moderate adherence are dying at a rate which is 2.3 times greater than patients with high adherence. The estimated 95 percent confidence interval for hazard ratio indicates that the hazard rate for patient with moderate adherence could be as much as 4 times higher than or as low as 1.2 times higher than patients who are high adherent. HIV positive patients who are low adherent to ART are dying at a rate which is 4.7 times greater than patients who are high adherent to ART. The 95 percent confidence interval for hazard ratio of low adherence level is 2 to 11 . This suggests those patient who are low adhere to ART may die as much as 11 times higher than or as little as 2 times higher than patients who are high adherent to ART. Moreover, to see the significant difference between patients who are low adherent and moderate adherent, we conduct the hypothesis test. Let $\beta_{2}$ and $\beta_{3}$ be the coefficient of low adherence level and moderate adherence level. The hypothesis test that we are going to test is:

$$
\text { Ho: } \beta_{2}=\beta_{3} \text { versus } H_{1}: \beta_{2} \neq \beta_{3}
$$

The test statistic is:

$$
\begin{gathered}
Z=\frac{\hat{\beta}_{3}-\hat{\beta}_{2}}{s \cdot e\left(\hat{\beta}_{3}-\hat{\beta}_{2}\right)} \\
Z=\frac{0.850-1.567}{0.483}=1.484
\end{gathered}
$$

The absolute value of $\mathrm{Z}$ is less than $\mathrm{Z}_{0.025}=1.96$, which means we fail to reject the null hypothesis that the two coefficients are equal and conclude that the death rate between the patients who are low adhere and high adhere may not be different at 5\% significance level.

The reference category for covariate 'condom use during sexual intercourse' is "never". The estimated hazard ratio for category "sometimes" is 0.279 . This means that a patient who had sometimes used a condom during sexual intercourse, are dying at a rate which is $72 \%$ less than the people who had never used condom during sexual intercourse. The rate could be as much as 91 percent lower to 8 percent lower. The estimated hazard ratio for patients who had used condom mostly was 0.116 . Patients who had used condom mostly are dying at a rate which is $88 \%$ smaller than patients who had never used condom. There was no the significance difference in hazard ratio between category "sometimes" and "mostly".

The reference category for "WHO stage" of HIV/AIDS patients on ART is "stage 4". The estimated hazard ratio for "WHO stage I" is 0.28 . The interpretation is that patients who had baseline WHO stage I are dying at a rate which is 72 percent lower than patients who had baseline WHO stage IV. The 95 percent confidence interval for hazard rate could actually be as much as 92 percent lower to only 11 percent lower. The estimated hazard ratio of "WHO stage II" is 0.3 , which implies that the death rate of WHO stage II patients is 0.3 times the death rate of WHO stage IV patients. The $95 \%$ confidence interval is that the hazard ratio could be as large as 0.14 and as low as 0.66 . WHO stage III patients are dying at a rate which is 0.4 times WHO stage IV patients. WHO stage III patients are dying at a rate which is 60 percent lower than patients in WHO stage IV. The $95 \%$ confidence interval is that the hazard rate could actually be as large as 82 percent lower than or as low as 28 percent lower than WHO stage IV patients.

Now, we see whether there is statistical difference between WHO stage II and WHO stage III patients. Let $\beta_{2}$ and $\beta_{3}$ be the coefficients of WHO stage II and WHO stage III, the hypothesis test that we are going to test is as follows:

$$
\text { Ho: } \beta_{2}=\beta_{3} \text { versus } H_{1}: \beta_{2} \neq \beta_{3}
$$

The test statistics is:

$$
\begin{gathered}
Z=\frac{\hat{\beta}_{2}-\hat{\beta}_{3}}{s \cdot e\left(\hat{\beta}_{2}-\hat{\beta}_{3}\right)} \\
Z=\frac{-1.165-(-1.042)}{0.521}=-0.236
\end{gathered}
$$

The absolute value of $\mathrm{Z}$ is less than $Z_{0.025}=1.96$, which means we fail to reject the null hypothesis that the two coefficients are equal. We conclude that the death rate between WHO stage II and WHO stage III patients is not significantly different at $5 \%$ significance level. The $\mathrm{Z}$ value that tests WHO stage I to WHO stage II and WHO stage III is -0.35 and -0.17 , respectively. The absolute value is less than the critical value $\left(Z_{0.025}=1.96\right)$. Therefore, we don't reject the null hypothesis that says there is no statistically difference between those coefficients.

Marital status is found significant at 5 percent significance level in the final model. As we have seen in the model, married patients are dying at a hazard rate which is 57 percent lower than single patients. The 95\% confidence interval of hazard ratio is as large as 0.84 and as small as 0.22 . The divorced patients are dying at the hazard rate which is 51 percent lower than single patient. The $95 \%$ confidence interval of hazard ratio is as large as 0.976 and as small as 0.247 . Now we see whether significance difference between married and divorced hazard rate. Let $\beta_{2}$ and $\beta_{3}$ be the coefficients of married and divorce respectively.

$$
\text { Ho: } \beta_{2}=\beta_{3} \text { versus } H_{1}: \beta_{2} \neq \beta_{3}
$$

The test statistics is:

$$
\begin{gathered}
Z=\frac{\hat{\beta}_{2}-\hat{\beta}_{3}}{s \cdot e\left(\hat{\beta}_{2}-\hat{\beta}_{3}\right)} \\
Z=\frac{(-0.84)-(-0.710)}{0.4823}=-0.269
\end{gathered}
$$

The absolute value is less than $Z_{0.025}=1.96$. We fall to reject the null hypothesis that the two coefficients are equal at 5 percent significance level. 
HIV/AIDS patients "who are not user of alcohol" is taken as a reference category in this study. The estimated hazard ratio for patients who were alcohol user is 2.5. It suggests, The HIV/AIDS patients who were alcohol user are dying at a rate which is 2.5 times higher than patients who were not alcohol user. The estimated $95 \%$ confidence interval of hazard ratio is $(1.4,4.5)$, which suggests that the value could be as much as 4.5 higher and as little as 1.4 higher.

Table 2. Cox Proportional Hazard Model to HIV Positive Adult Patients at Hawassa and Dilla University Referral Hospital ART unit.

\begin{tabular}{|c|c|c|c|c|c|c|c|}
\hline Variable & $\widehat{\boldsymbol{\beta}}$ & SE & Wald & DF & P-value & $\operatorname{Exp}(\widehat{\beta})$ & 95\% CI for $\operatorname{Exp}(\widehat{\beta})$ \\
\hline Age & 0.049 & 0.014 & 11.583 & 1 & 0.001 & 1.050 & $(1.020,1.079)$ \\
\hline CD4 & -0.004 & 0.001 & 10.603 & 1 & 0.001 & 0.996 & $(0.994,0.998)$ \\
\hline Condom Use (Never R) & & & 6.350 & 2 & 0.042 & & \\
\hline Sometimes & -1.275 & 0.611 & 4.355 & 1 & 0.037 & 0.279 & $(0.084,0.925)$ \\
\hline Mostly & -2.157 & 1.018 & 4.492 & 1 & 0.034 & 0.116 & $(0.016,0.850)$ \\
\hline Marital status (Single R) & & & 10.251 & 3 & 0.017 & & \\
\hline Married & -0.840 & 0.342 & 6.049 & 1 & 0.014 & 0.432 & $(0.221,0.843)$ \\
\hline Widowed & -0.649 & 0.422 & 2.363 & 1 & 0.124 & 0.522 & $(0.228,1.196)$ \\
\hline Functional (Work R) & & & 13.040 & 2 & 0.001 & & \\
\hline Bedridden & 1.082 & 0.520 & 4.33 & 1 & 0.037 & 2.951 & $(1.065,8.170)$ \\
\hline Ambulatory & 1.079 & 0.301 & 12.734 & 1 & 0.000 & 2.941 & $(1.624,5.31)$ \\
\hline Alcohol Use (NO R) & 0.925 & 0.301 & 9.452 & 1 & 0.002 & 2.521 & $(1.400,4.537)$ \\
\hline WHO stage (IV R) & & & 12.041 & 3 & 0.007 & & \\
\hline I & -1.284 & 0.598 & 4.611 & 1 & 0.032 & 0.277 & $(0.086,0.894)$ \\
\hline II & -1.165 & 0.383 & 9.266 & 1 & 0.002 & 0.312 & $(0.147,0.660)$ \\
\hline Adherence level (High R) & & & 16.499 & 2 & 0.000 & & \\
\hline Low & 1.567 & 0.443 & 12.510 & 1 & 0.000 & 4.794 & $(2.011,11.42)$ \\
\hline Moderate & 0.850 & 0.314 & 7.311 & 1 & 0.007 & 2.339 & $(1.263,4.330)$ \\
\hline
\end{tabular}

\subsubsection{Discussion of Survival Analysis Results}

Although many medications are used to treat human immunodeficiency virus (HIV) infection, none can cure HIV/AIDS disease. Here, the Cox proportional hazard model applied to assess the determinant factors of survival time of HIV/AIDS patients.

This 5 year retrospective cohort study of HIV/AIDS patients at Hawassa and Dilla University Referral Hospital ART center gives an insight into survival time pattern of patients and its determinants in a hospital setting in Ethiopia. In this cohort $14.5 \%$ of the patients died and most of the death occurred within the first 5 months. The overall death rate was smaller when compared to others study in Africa in which it was shown to be $29.7 \%$ in Tanzania [1] and $23.0 \%$ in Cameron [10]. Most of the patient died during the first 12 months. The high mortality in the first few months was similar to other studies in Africa [1] and [10], including Ethiopia [2]. There was not a significant difference between male and female in survivorship function.

Age of the patient found significant predicator for survival time of the patient. When we compare the survivorship function for categorized age groups, patients in age group 15 up to 27 lived longer than other groups. The smallest survivorship function is estimated for patients who have been more than 37 years old. Study comparable to this in Africa [1] shown that the survival curve for patients who had age greater than 40 years consistently went below than patients who had age less or equal to 39 years. And, the result showed that there was significant survival difference between those age groups. This result consistent with study in Addis Ababa [17] that patients who were in aged group (age above 60 years) lived smaller than patients found below that age groups. In comparable study in Singapore [3], the estimated hazard ratio of age was 1.02 (adjusted $\mathrm{HR}=1.2, \mathrm{CI}=101$ $1.03, \mathrm{P}$ value $=0.006$ ). Age is not significant predicator of HIV/AIDS patient's survival time in some paper [1, 2, 3, 19]. In this study, the HIV /AIDS patient whose age growth by 5 year are dying at a rate which is higher $28 \%$. In other word, for 5 year growth in patient age, the hazard of death is 1.28.

In this study, survivorship function is significantly different in WHO stage of HIV/AIDS patient, this result similar with other study in Ethiopia $[2,3,19]$ and some other country in Africa $[1,10]$. WHO stage IV was found predicator of death in this study, this supports (similar to) the study that done in African country [4, 11, 15]. WHO stage IV patients had less survival experience. The estimated hazard ratio for WHO stage I, WHO stage II and WHO stage III is $0.28(\mathrm{HR}=$ $0.28, \mathrm{CI}=0.08-0.89), 0.31(\mathrm{HR}=0.31, \mathrm{CI}=0.14-0.66)$ and $0.35(\mathrm{HR}=0.35, \mathrm{CI}=0.17-0.71)$, respectively. This result reveals patients in WHO stage I, stage II and stage III are dying at a rate which is $72 \%, 69 \%$, and $65 \%$ lower than WHO stage IV patients, respectively. In comparable study at Bahir-dar Feleg Hiwot Referral Hospital, in Ethiopia [19], the estimated hazard ratio of WHO stage I, WHO stage II and WHO stage III patients is $75 \%(\mathrm{HR}=0.253, \mathrm{CI}=0.11-0.76)$, $73 \% \quad(\mathrm{HR}=0.267, \quad \mathrm{CI}=0.10-0.75) \quad$ and $22 \% \quad(\mathrm{HR}=0.781$, $\mathrm{CI}=0.48-1.26)$, respectively .

Marital status found in this research is significant predicator of hazard of death in HIV/AIDS patients. In the cohort, the median survival time for single, married, divorce and widowed patients is 33, 33, 35 and 39 respectively. Married and divorced patients lived more than 7 month on average than single patients and more than 2 month on average than widowed patients. In a study, Hawassa Referral Hospital [18], marital status has significant association with 
survival time of patients ( $\mathrm{p}$ value $<0.005$ ) for 1110 sample patients. Moreover, female patients had favorable survival experience than others. Comparable study in South Africa [11] found that marital status significantly predicator of mortality in HIV patients within antiretroviral therapy (ART). This result is consistent to our findings.

\subsubsection{Clinical Progression of AIDS Disease}

The possible transition of the immunological stages of adult HIV positive patient going on in Hawassa and Dilla University Referral Hospital is presented Figure 3. This study considered that an infected patient can move among the following immunological marker stages related to CD4 count: stage I $\left(C D 4>500\right.$ cells $\left./ \mathrm{mm}^{3}\right)$, stage II (350 cells / $\mathrm{mm}^{3}<C D 4 \leq 500 \mathrm{cells} / \mathrm{mm}^{3}$ ), state III (200 cells / $\left.\mathrm{mm}^{3}<C D 4 \leq 350 \mathrm{cells} / \mathrm{mm}^{3}\right)$ and state IV (CD4 $\leq$ 200 cells $/ \mathrm{mm}^{3}$ ). Moreover, the patient death added as an absorbing state. Among four state of the disease, 20 transitions came out. Patient started the treatment under any state has a possibility to reach any other state. If there is an improvement on CD4 count, the patient has a recovery from the state where he is. Unfortunately, patient may go to death from any working state. The transition of the patient in different state occurs at any time. Here, we use the word 'state' and 'stage' interchangeably.

The transition frequency matrix of the embedded Markov chain formed by the possible states of the process is shown in Table 3. In the followed up period, 128, 176, 270 and 449 transitions had already been from state I, II, III, and IV, respectively. The number of death observed from the successive working state i.e. I, II, III, and IV was 3, 4, 15, and 40 , respectively. The number of death from state IV is as high compared to others. Seven patients transited at state IV from state I while 184 left the state IV and then come to state I. State II and III seem to be relatively more transitive states regarding to the number of observed transition frequency.

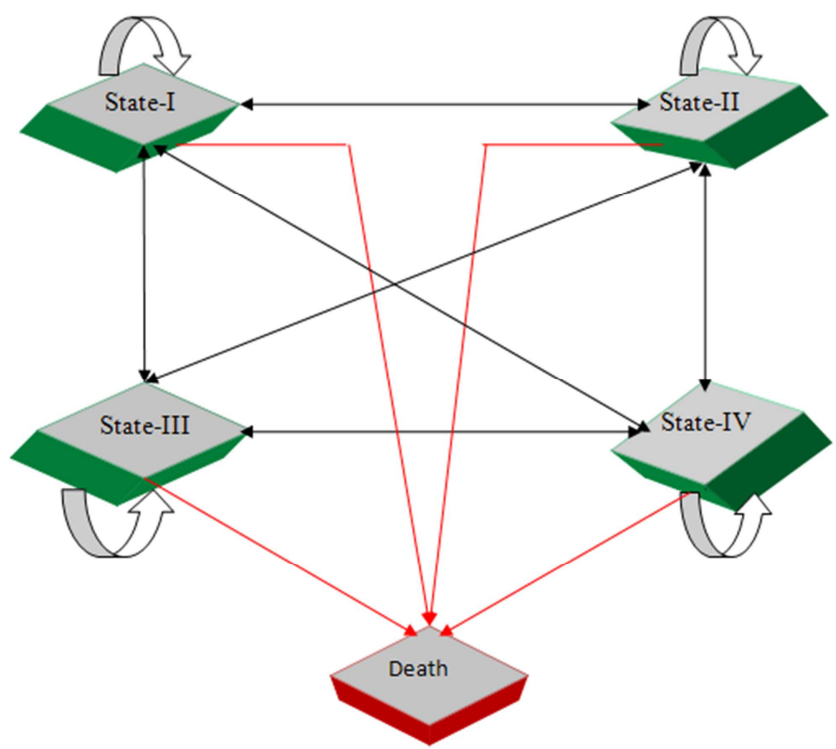

Figure 3. The Model of the Immunological Stages of Adult HIV/AIDS Patient goes on (at Hawassa and Dilla University Referral Hospital ART Center, 2007-2012).
A patient in the state I, II, III, and IV dies with probability $0.23,0.22,0.55$ and 0.89 , respectively in Table 4 . A patient from state I, II and III enters to state IV with probability 0.55 , 0.80 and 0.7 , respectively. Patients show improvement from state IV to state III, state II and state I with probability 0.22 , 0.16 and 0.11 , respectively. Patients show improvement from state IV to III, from state III to II and from state II to I with probability $0.22,0.18$ and 0.21 , respectively. A patient from state I enter to state IV with probability 0.54 .

Table 3. Transition Frequency Matrix for HIV Positive Adult Patients at Hawassa and Dilla University Referral Hospital ART center (Year: 20072012).

\begin{tabular}{llllll}
\hline State & S-I & S-II & S-III & S-IV & Death \\
\hline S-I & 96 & 12 & 10 & 7 & 3 \\
S-II & 37 & 104 & 17 & 14 & 4 \\
S-III & 66 & 51 & 120 & 18 & 15 \\
S-IV & 50 & 75 & 100 & 184 & 40 \\
\hline
\end{tabular}

Table 4. Estimates of the Transition Probability Matrix of the Embedded Markov Chain (At Hawassa and Dilla University Referral Hospital ART center, during 2007-2012).

\begin{tabular}{llllll}
\hline State & S-I & S-II & S-III & S-IV & Death \\
\hline S-I & 0.7500 & 0.2000 & 0.0781 & 0.0547 & 0.0234 \\
S-II & 0.2102 & 0.5909 & 0.0966 & 0.0795 & 0.0227 \\
S-III & 0.2444 & 0.1889 & 0.4444 & 0.0667 & 0.0556 \\
S-IV & 0.1114 & 0.1670 & 0.2227 & 0.4098 & 0.0891 \\
Death & 0.0000 & 0.0000 & 0.0000 & 0.0000 & 1.0000 \\
\hline
\end{tabular}

\subsubsection{Prediction of Clinical AIDS Disease Progression in Individual Patient}

The probability that a patient starting from stage $i \in$ $\{I, I I, I I I, I V\}$ at time 0 enters state $j \in\{I, I I, I I I, I V\}$ after month $\mathrm{t}$ is plotted in Figure 4 Figure $4 \mathrm{~A}$ shows that the probability that a patient starting from stage I at time 0 , after month $\mathrm{t}$ enters to stage $j \in\{I, I I, I I I, I V\}$. The probability of remaining in its stage is high as compared to others till 168 months. The probability of a patient starting from stage I at time zero enters to stage $j \in\{I, I I, I I I, I V\}$ and Death after 168 months, estimated to be $0.36,0.26,0.22,0.08$ and 0.05 , respectively. The conditional probability that a patient starting from stage I at time zero, enters to stage $j \in$ $\{I, I I, I I I, I V\}$ and Death after 200 month, are estimated to be $0.29,0.27,0.09,0.05$ and 0.28 , respectively.

The conditional probability that a patient starting from stage II at time zero enters after month to stage $j \in$ $\{I, I I, I I I, I V\}$ is plotted in Figure 4B. As we can see from this plot, the probability to stay in same stage II is higher compared to others till month 52 and then more likely goes to stage I after month 48. The conditional probability that a patient from stage II at time zero will reach stage $\mathrm{j} \in$ $\{$ I, II, III, IV $\}$ and Death after month 120 , are estimated to be $0.49,0.26,0.16,0.08$ and 0.04 respectively. The probability of a patient starting from stage IV at time zero enters to stage I, II, III and IV after 200 months with probabilities 0.32 , $0.80,0.16$ and 0.23 respectively.

Figure $4 \mathrm{C}$ presents the conditional probability that a 
patient who is in stage III will move to stage $\mathrm{j} \in$ $\{$ I, II, III, IV $\}$ and Death after t months. Eighteen month later, patients leave stage III with probability of 0.3 . The stage I seem more transient stage for a patient who come from stage III at time zero. The probability of a patient from stage III at time zero will move to stage $j \in\{I, I I, I I I, I V\}$ and Death after 200 months, estimated as 0.3, 0.22, 0.08, 0.04 and 0.29 respectively. After 48 months, probability of a patient to move from stage III to stage IV is very small compared to other stages. The conditional probability of entering stage $j \in$ $\{I, I I, I I I, I V\}$ and Death from stage IV after $\mathrm{t}$ months is plotted in Figure 4D. As we can see from this graph, stage II and stage III seem more transient stage. Two hundred month later, patients make recovery from stage IV through going to stage I with probability 0.27 . The conditional probability that a patient starting from stage IV at time zero, die 200 months later is 0.4 .

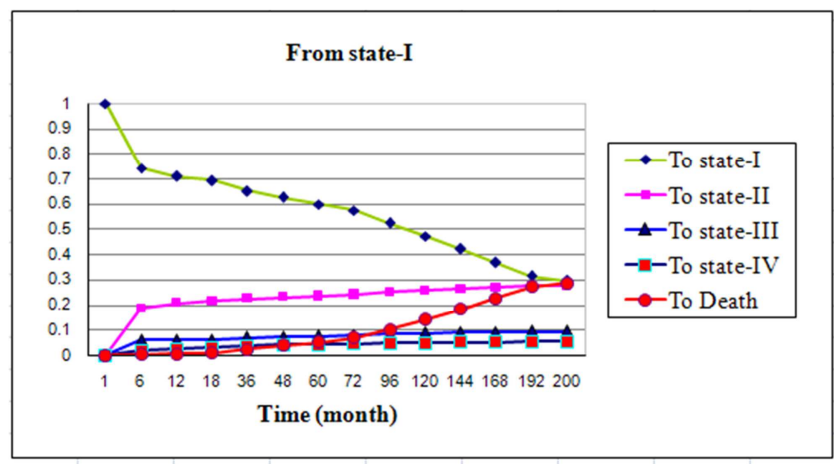

A

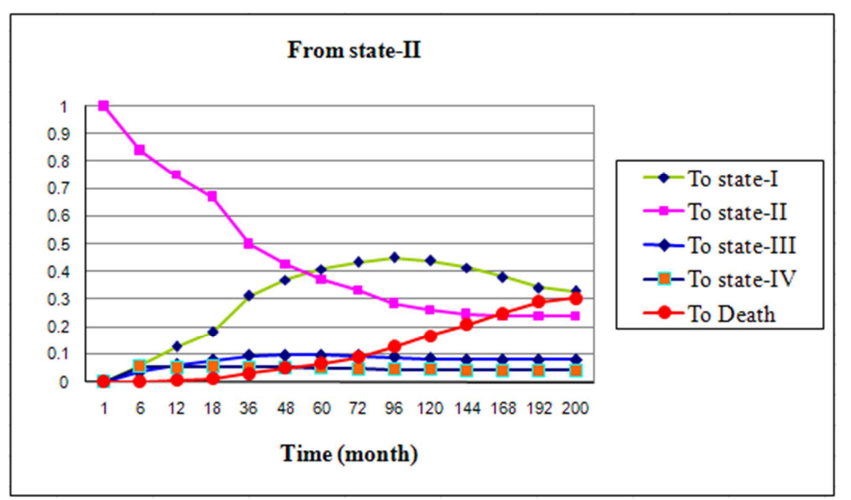

B

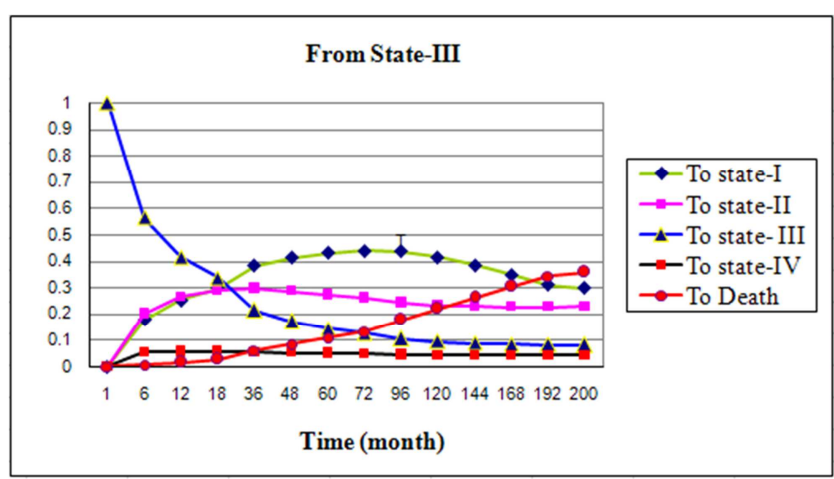

C

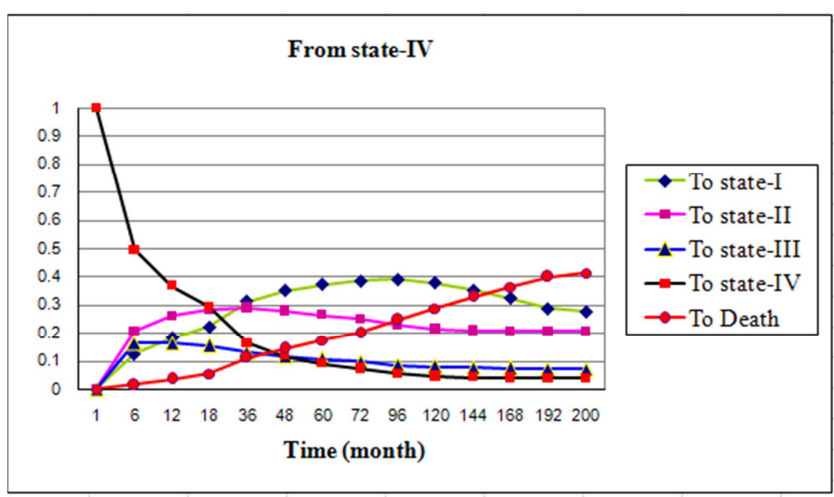

D

Figure 4. Conditional Probabilities for Each Stage.

\subsubsection{Discussion for AIDS Disease Progression Analysis Results}

The HIV/AIDS evolution model considered in this study relates to a 'macroscopic' view of the disease process and it is based on the CD4 cell count measurement. In this study we considered that an infected patient can move among the following immunological states related to CD4 cell counts: state I $\left(C D 4>500\right.$ cells $\left./ \mathrm{mm}^{3}\right)$, state II $\left(350\right.$ cells $/ \mathrm{mm}^{3}<$ CD4 $\leq 500$ cells $\left./ \mathrm{mm}^{3}\right)$, state III $\left(200\right.$ cells $/ \mathrm{mm}^{3}<$ CD4 $\leq 350$ cells $\left./ \mathrm{mm}^{3}\right)$ and state IV (CD4 $\leq 200 \mathrm{cells} /$ $\mathrm{mm}^{3}$ ). The patient death is considered as an absorbing state. Patient who started the treatment under any state has a possibility to reach any other state. If the medical tests show an improvement in the CD4 cell count, patient has recovery from the stage where he is.

The semi-markov model is a useful tool to predict the clinical progression of a disease. It consists mainly of computing the probability of a patient being into one of the possible stages of the disease for a certain time and the probability that the patient might survive for a time t. The most important property of semi-Markov processes is that they enable us to consider not only the randomness in the different states in which the infection can evolve into but also the randomness of the time spent in each state.

In this study we are able to see the conditional probability that a patient leaves own baseline state or a conditional probability of a patient remaining in the given stage after time period t. Moreover, the conditional probability that the failure of the patient occurs at month $\mathrm{k}$, given that the patient has survived until specified previous month is estimated. The conditional probability that the death of a patient is at six years, given that a patient survived up to four years, for stage I, stage II, stage III and stage IV, estimated as $0.024,0.032,0.04$ and 0.05 respectively. The probability of remaining in the starting stage after 72 month for patients from stage II, stage III and stage III is approximately zero. This suggests that almost all patients transit to other stage 72 month later. Comparable study by [19] indicated that a patient at stage I, stage II, stage III or stage IV was highly likely to leave the same state to other states after month 78 . The probability of a patient starting from stage I, II, III and IV, dies after 120 
months with probability $0.14,0.17,0.22$ and 0.28 respectively. The probability that a patient being at time zero in stage I, II, III and IV dies after 200 months are estimated to be $0.28,0.30,0.35$ and 0.41 respectively. Comparable study by [19] indicated that the probability of a patient starting from stage I, II, III and IV dies after 200 months are estimated to be $0.39,0.44,0.48$ and 0.54 , respectively.

\section{Conclusions}

We sought to determine the factors prior to ART initiation that were most predictive of survival time in HIV/AIDS patients after initiation ART in Hawassa and Dilla University Referral Hospital, Ethiopia. In the cohort, age, marital status, condom use during sexual intercourse, alcohol use, functional status, CD4 cell count, WHO stage and adherence level are statistically significantly related to survival time of HIV/AIDS patients.

Patients who had been in age group 15 up to 27 years lived longer than patients who had been in age above 27 years. Marital status was statistically significant predicator of survival time in HIV/AIDS patients. In the study, married patients had favorable survival experience than other patients. Condom use mostly during sexual intercourse is associated with lower hazard of death. Patients who were using condom mostly have had better survival experience. Alcohol use was associated with lower survival experience.

HIV/AIDS patients who could 'work' or not in bed had favorable survival experience than others. Bedridden patients had higher hazard ratio than ambulatory patients. CD4 cell count was also a significant predicator of survival time in HIV/AIDS patients. The increment of patient CD4 cell count had a significant effect on favorable survival time. WHO stage IV patients had low survival experience than others. Low adherence level was associated with high hazard of death. Patients who were high adherent to ARV had a significant effect on favorable survival experience.

The interaction effect of variables was assessed and no interaction had a significant effect in survival time of HIV/AIDS patients. The significance within variable levels was assessed. The result indicated that there was no statistically significance difference within categories.

The discrete time homogeneous semi-markov model was used to see clinical progression of HIV/AIDS disease. Semimarkov reliability model was employed to obtain the probability that a patient was alive from time 0 at any stage to a time t. Failure rate of discrete time semi-markov model was addressed to obtain the conditional probability that the death of patient occurs at time $\mathrm{k}$, given that a patient had survived until time k-1.

The conditional probability of being in the next worse stage of the disease rises rapidly in the initial time and followed by a peak but gradually decline. The survival probability of a patient depends on the immunological marker of CD4 cell count. Throughout time probability of death was higher for patients who had started from state IV. However, the result had shown that probability of improving the clinical progression of AIDS disease by recovering the patients CD4 cell count.

\section{Acknowledgments}

I also appreciate the financial support of the Office of Research, Dissemination and Community Services of Dilla University, during the research work.

\section{References}

[1] Asgeir, J., N. Ezra, J. Bernand, S. Leiv, I. Mecky, A. E. Henry, G. G. Svein, N.B. John, (2008). Predicators of Mortality in HIV Infected Patients Starting ART in a Rural Hospital Tanzania. Journal of BMC Infectious Disease. 8:52.

[2] Andinet, W. (2009). Pattern and Determinants of Survival in Adult HIV patients on ART, Ethiopia. Umea International School of Public Health, Umea University.

[3] Birtukan, T. (2010). Predictors of Early Death in HIV Positive Individuals Enrolled for Chronic HIV Care in Jimma Zone, South Western Ethiopia. Masters Thesis, Addis Abeba University. Addis Abeba.

[4] Etard, J. F., Ndiaye I, Thierry M, Gueye NF, Gueye PM, Laniece I,Dieng AB, Diouf A, Laurent C, Mboup S, Sow PS, Delaporte E (2006). Mortality and Causes of Death in Adults Receiving Highly Active ART in Senegal. A 7-year cohort study, 20:1181-1189.

[5] Federal Ministry of Health (2006). AIDS in Ethiopia, Sixth Report, Addis Ababa, Ethiopia, pp. 150.

[6] Federal Ministry of Health (2008). AIDS in Ethiopia, Report, Addis Ababa, Ethiopia, pp. 180.

[7] Federal Ministry of Health (2007). Antiretroviral Treatment for Adult and Adolescence. Addis Abeba, Ethiopia.

[8] Federal Ministry of Health (2010). Antiretroviral Therapy in Ethiopia. Addis Abeba, Ethiopia.

[9] Giuseppe, D., Amico, G. D., A. Girolamo, J. Janssen, S. Iacobelli, N. Tinari, R. Manca (2007). A Stochastic Model for the HIV/AIDS Dynamic Evolution. Mathematical Problems in Engineering, ART. ID 65636, Vol. 20, pp. 14.

[10] Isidore, S., S. Mohamadou, M.S. Anne, M. Joris, B. Marleen, (2009). Determinants of Survival in AIDS Patients on ART in a Rural Center in the Far-North Province, Cameron. Journal of Tropical Medicine and International Health. 14 (1): 3643.

[11] Lawn, S. D., Myer L, Harling G, Orrell C, Bekker LG, Wood R (2006). Determinants of Mortality and Non-death Losses from an ART service in South Africa: Implications for Program Evaluation. Journal of Clinical Infectious Disease. 43: 770-776.

[12] Michael, A. T. (2010). Antiretroviral Therapy. MPH, USA.

[13] Mogiyana, T., D. Mariw and C. Tiana (2009). Mortality and Causes of Death in HIV Positive Patients Receiving Antiretroviral Therapy at Tshepang Clinic in Doctor George Mukhari Hospital. 118 (10). 
[14] Nuredin Ibrahim (2007). Education of Factor Affecting the Chance of Survival/Death Status Among HIV Positive People Under the Antiretroviral Treatment Program: The Case of Adama Referral Hospital, M.Sc. Thesis, Addis Ababa University, Ethiopia.

[15] Stringer, J.S., Zulu I, Levy J, Stringer EM, Mwango A, Bulterys M, Saag MS, Marlink RG, Mwinga A,Ellerbrock TV, Sinkala M (2006). Rapid Scale-up of ART at Primary Care Sites in Zambia: Feasibility and Early Outcomes. JAMA, 296: 782-793.

[16] United Nations Program on AIDS (2006). Report on the Global HIV/ AIDS Epidemic. Geneva, 11: 85-97.
[17] United Nation Program on AIDS (2010). Global Health Report (http:/www.globalhealthre Porting.org/diseaseinfo.25, June, 2011).

[18] Tigest Assefa (2007). Survival Analysis on HIV/AIDS Patients at Tekur Ambesa Hospital. M.Sc. Thesis, Addis Ababa, Ethiopia.

[19] Zelalem Getahun (2010). Statistical Modelling of HIV/ AIDS Progression and Survival of AIDS Patients. A Case Study of Bahir-Dar, Feleg-Hiwot Referral Hospital. M.Sc. Thesis. Hawassa University, Hawassa, Ethiopia.

[20] Zhang, X. (2007). HIV/AIDS Relative Survival Analysis. Master's Thesis Published in Georgia University. USA. 